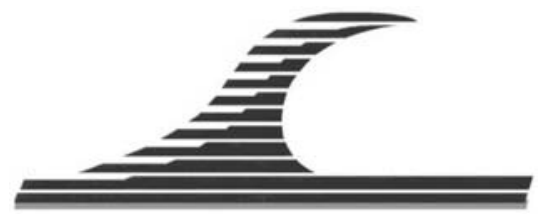

\title{
Analyse statistique des données obtenues sur les sédiments traités sur le site pilote de SEDI.MAR.D 83
}

\author{
Christelle BENOIT-BONNEMASON ${ }^{1}$, Fabienne SEBY ${ }^{1}$, \\ Jean-Christophe TURLOT ${ }^{2}$, Mickael JOURDAN ${ }^{3}$, Claude ALZIEU ${ }^{3}$, \\ Jean-Luc AQUA ${ }^{3}$, Laurent SANNIER ${ }^{3}$, Olivier F.X. DONARD ${ }^{4}$
}

1. UT2A, Hélioparc Pau-Pyrénées, 2 Av du Pdt Angot, 64053 Pau cedex 9, France. christelle.benoit@univ-pau.fr

2. LMA/ UMR CNRS 5142, UPPA, Av de l'Université, 64000 Pau, France

3. Conseil Général du Var, Direction des Ports, 390 Av des Lices, BP1303, 83076 Toulon cedex, France.

4. LCABIE / IPREM, UMR CNRS/UPPA 5254, Hélioparc Pau-Pyrénées, 2 Av du Pdt Angot, 64053 Pau cedex 9, France.

\section{Résumé :}

Le projet SEDI.MAR.D 83 avait pour objectif de proposer différentes techniques de traitements afin d'optimiser la gestion à terre de sédiments marins dragués provenant de ports méditerranéens. Dans ce but, une caractérisation physico-chimique des sédiments a été réalisée sur l'échantillon brut initial mais également à la fin de chaque traitement appliqué. Une base de données importante a ainsi été obtenue pour différents contaminants ainsi que des données complémentaires sur les propriétés physiques et les nutriments. L'objectif de ce travail est d'utiliser des outils statistiques pour hiérarchiser les différentes filières de traitement et établir des corrélations entre les différents paramètres géochimiques.

Il a été montré que deux ports (Arsenal et Guilvinec) se distinguent des autres ports par une pollution importante. Parmi les différents traitements appliqués, le compostage s'avère particulièrement intéressant pour les composés organiques et les composés organométalliques. Pour ces mêmes composés, le traitement par calcination engendre également un abattement des concentrations. En revanche, pour les métaux comme le cuivre, aucun traitement n'apparaît présenter un effet positif sur la décontamination. Par contre, la phosphatation et la calcination (procédé NOVOSOL®) semblent favoriser l'inertage du sédiment par piégeage du cuivre dans la masse sédimentaire.

Soumis le 26 février 2009, accepté le 12 février 2010, en ligne le 2 juillet 2012.

La seule version examinée est celle écrite en français. La ou les autres versions n'étant pas examinées par le comité de rédaction de la revue, sont donc publiées sous l'entière responsabilité du ou des auteurs.

Pour citer cet article :

BENOIT-BONNEMASON C., SEBY F., TURLOT J.-C., JOURDAN M., ALZIEU C., AQUA J.-L., SANNIER L., DONARD O.F.X. (2012). Analyse statistique des données obtenues sur les sédiments traités sur le site pilote de SEDI.MAR.D 83. Revue Paralia, Vol. 5, pp 3.1-3.16. 


\section{Introduction}

Le projet SEDI.MAR.D.83, mis en place par le conseil général du Var, a pour objectif de proposer une gestion à terre comme solution alternative à l'immersion des sédiments marins contaminés. Pour ce faire, différentes techniques de prétraitements et traitements ont été testées sur des sédiments portuaires dans le but de définir des filières de valorisation les plus adaptées (AQUA et al., 2009).

Un diagnostic initial des sédiments des différents ports concernés par le projet a permis de caractériser ces matériaux d'un point de vue physico-chimique selon les exigences de l'arrêté du 14 juin 2000 (JO n 184 du 10 août 2000) et de la circulaire afférente (BO $\mathrm{n}^{\circ}$ 2000/16). Différents types de contaminants métalliques (As, $\mathrm{Cd}, \mathrm{Cr}, \mathrm{Cu}, \mathrm{Hg}, \mathrm{Ni}, \mathrm{Pb}$, $\mathrm{Zn}$ ), organiques (PCB, HAP) et organométalliques (TBT) ont été recensés. Ces contaminants sont ubiquistes dans l'environnement marin et sont problématiques du fait de leur très grande toxicité lorsqu'il sont biodisponibles (BENOIT, 2005 ; HOCH, 2001, RIOS et al., 2007). D'autres paramètres permettant de caractériser le sédiment ont été recherchés tels que le carbone organique total (COT), la granulométrie, la matière sèche, le $\mathrm{pH}$, la densité, ainsi que la microbiologie (germes fécaux) et les nutriments (azote, phosphore total). L'ensemble de ces analyses a été réalisé sur les sédiments bruts et sur les sédiments traités. L'application d'un ou plusieurs traitements aura des incidences sur les caractéristiques physico-chimiques et/ou biologiques des sédiments (MAROT, 2007). Or toute modification de ces caractéristiques peut avoir des conséquences sur la biodisponibilité des contaminants et donc sur la toxicité du sédiment (RULKENS, 2005). En effet, la capacité des contaminants inorganiques à être mobilisés ou piégés dans le sédiment dépend de la forme chimique dans laquelle il se trouve, qui est en fonction des conditions biogéochimiques du milieu ( $\mathrm{pH}$, température, salinité, présence d'oxygène ou non, présence de matière organique, présence de minéraux, activité bactérienne) (LANDRUM \& ROBBINS, 1990 ; WRIGHT \& MASONU, 1998). Les traitements qui modifient les conditions physico-chimiques et/ou biologiques de la masse sédimentaire auront donc une influence directe sur la biodisponibilité des contaminants dans le sédiment et donc sur sa toxicité (CALMANO \& FORSTNER, 1996).

Dans cette étude, le suivi a porté sur le devenir des sédiments après l'application de différents traitements seuls ou combinés qui sont: le dessablage, la déshydratation, la phosphatation, le compostage et la calcination. A partir de l'ensemble des analyses, une base de données très riche a été constituée (près de 6000 analyses réalisées) qui renseignent sur les caractéristiques physico-chimiques de chaque sédiment avant (diagnostic initial), pendant et après traitement. C'est à partir de cette base de données que l'étude statistique a été menée. La première étape de cette étude a été d'élaborer une matrice statistique à partir des données les plus pertinentes obtenues par le projet. Seules les données provenant d'un même laboratoire et provenant de protocoles analytiques comparables ont été conservées. Dans une seconde étape, les données 
validées ont été utilisées pour une analyse statistique approfondie qui a permis d'une part de discriminer les différents sédiments entre-eux et, d'autre part, de hiérarchiser les différentes filières de valorisation et/ou de traitement et, enfin, d'établir des corrélations (Analyse en Composante Principale) entre les différents paramètres géochimiques, les principaux contaminants (PCB, HAP, métaux, organométaux) des sédiments portuaires et les différents traitements étudiés au cours du projet SEDI.MARD.83.

\section{Matériel et méthodes}

\subsection{Traitements des sédiments}

Après dragage de différents ports de la région PACA, les sédiments marins ont été transportés sur la plateforme de Brégaillon spécialement aménagée à cet effet. Une fois sur cette plate-forme, différents types de pré-traitements et/ou traitements ont été appliqués aux sédiments afin de réduire, voire d'éliminer ou de stabiliser leur charge polluante. Pour un même sédiment, les traitements vont être appliqués au sédiment brut tel qu'il arrive sur la plate-forme et à la fraction fine (la taille des particules inférieure à $63 \mu \mathrm{m})$. La fraction sableuse et la fraction fine sont obtenues après passage du sédiment brut au crible et à l'hydrocyclone. Chaque fraction sédimentaire subit ensuite un ou plusieurs traitements à l'issue duquel ou desquels des analyses physico-chimiques sont réalisées (SANNIER et al., 2009). Ces pré-traitements et traitements sont présentés figure 1 .

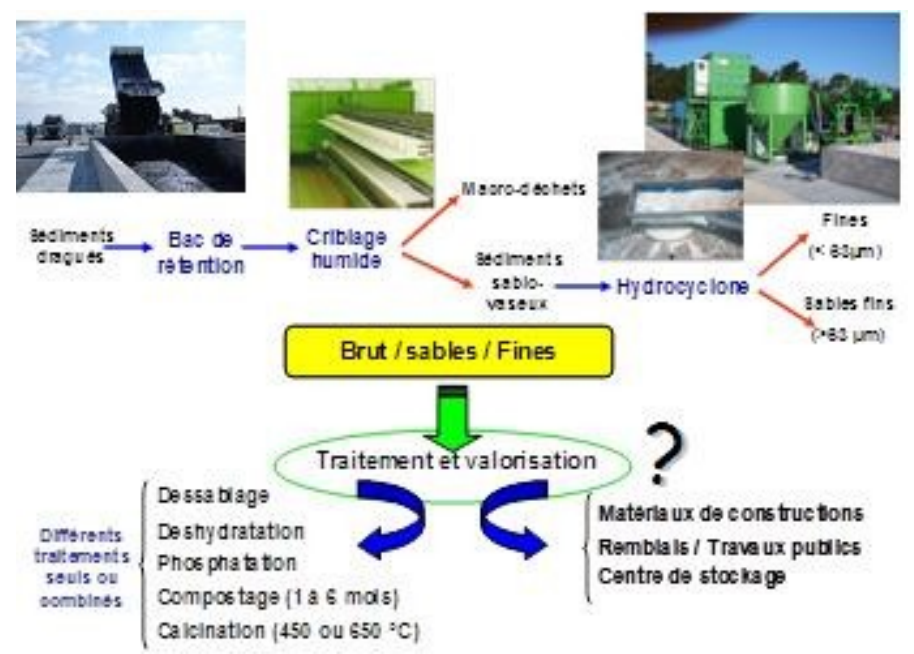

Figure 1. Synoptique de prise en charge des sédiments sur la plate-forme de Brégaillon

Le pré-traitement permet de modifier les caractéristiques physiques du sédiment (séparation granulométrique, déshydratation, etc.) et de conditionner le sédiment en vue d'un éventuel traitement (DELORT \& GRODEMANGE, 2006). Le traitement va permettre de modifier la nature et la dangerosité intrinsèque du sédiment selon 
différents mécanismes physiques, chimiques et biologiques. Il existe une large variété de traitements pouvant être appliqués au sédiment dans le but d'éliminer les contaminants (traitements thermique ou physique), de les dégrader (traitement chimique et/ou biologique), ou bien de les piéger dans la masse sédimentaire (principe de l'inertage) (RULKENS, 2005). Les spécificités des traitements sont rappelées dans le tableau 1.

Tableau 1. Spécificité des traitements et pré-traitements (GAN et al., 2009 ; RULKENS, 2005 ; VERMEULEN, 2003 ; QINGMAN et al., 2003)

\begin{tabular}{|c|c|c|c|}
\hline & Objectifs & Techniques & Intérêts \\
\hline Dessablage & $\begin{array}{l}\text { Séparer la fraction } \\
\text { sableuse de la } \\
\text { fraction fine } \\
(>63 \mu \mathrm{m})\end{array}$ & $\begin{array}{l}\text { Bassin de } \\
\text { décantation } \\
\text { Hydrocyclone }\end{array}$ & $\begin{array}{l}\text { Réduire le volume du sédiment } \\
\text { à traiter et récupérer le sable } \\
\text { pour le valoriser (BTP, } \\
\text { remblais, travaux routiers, } \\
\text { etc.) }\end{array}$ \\
\hline Déshydratation & $\begin{array}{l}\text { Assécher le } \\
\text { sédiment }\end{array}$ & $\begin{array}{l}\text { Décantation } \\
\text { Egouttage par } \\
\text { géotubes }{ }^{\circledR} \\
\text { Ajout de chaux }\end{array}$ & $\begin{array}{l}\text { Atteindre une siccité }>30 \% \\
\text { permettant la valorisation ou } \\
\text { la mise en décharge du } \\
\text { sédiment }\end{array}$ \\
\hline Compostage & $\begin{array}{l}\text { Faire diminuer la } \\
\text { charge polluante } \\
\text { par dégradation } \\
\text { bactérienne en } \\
\text { milieu aérobie }\end{array}$ & $\begin{array}{l}\text { Stockage à terre } \\
\text { avec retournement } \\
\text { mécanique } \\
\text { régulier pour } \\
\text { oxygéner le } \\
\text { sédiment }\end{array}$ & $\begin{array}{l}\text { Dégrader certains polluants en } \\
\text { particulier les composés } \\
\text { organiques (HAP, PCB, TBT) } \\
\text { en favorisant l'activité } \\
\text { bactérienne en milieu aérobie }\end{array}$ \\
\hline Phosphatation & $\begin{array}{l}\text { Stabiliser le } \\
\text { sédiment }\end{array}$ & $\begin{array}{l}\text { Ajout d'acide } \\
\text { phosphorique (2 à } \\
3 \% \text { en masse) }\end{array}$ & $\begin{array}{l}\text { Piéger les métaux dans des } \\
\text { phosphates calciques formés } \\
\text { par réaction entre l'acide } \\
\text { phosphorique et les carbonates } \\
\text { de calcium }\end{array}$ \\
\hline Calcination & $\begin{array}{l}\text { Dégrader la matière } \\
\text { organique et les } \\
\text { contaminants } \\
\text { organiques et, } \\
\text { piéger les métaux } \\
\text { dans la matrice } \\
\text { sédimentaire }\end{array}$ & $\begin{array}{l}\text { Incinérateur, four } \\
\text { ou unité de } \\
\text { traitement } \\
\text { apparentée } \\
\text { permettant } \\
\text { d'atteindre de très } \\
\text { hautes } \\
\text { températures }\end{array}$ & $\begin{array}{l}\text { Généralement couplée à un } \\
\text { pré-traitement: } \\
\text { - à la déshydratation: pour } \\
\text { réduire le volume du matériau } \\
\text { et donc le coût du traitement } \\
\text { - à la phosphatation: (procédé } \\
\left.\text { NOVOSOL }{ }^{\circledR}\right) \text { pour inerter le } \\
\text { matériau en piégeant les } \\
\text { métaux }\end{array}$ \\
\hline
\end{tabular}




\subsection{Données obtenues dans le cadre de SEDI.MAR.D 83}

Des analyses physico-chimiques ont été réalisées sur les sédiments, toute fraction confondue, ce qui représente près de 6000 données. Les différents types d'analyses sont les propriétés physico-chimiques (siccité, densité, $\mathrm{pH}$, chlorures, granulométrie, COT), les nutriments (phosphore total, azote kejdhal), les contaminants inorganiques (Al, As, $\mathrm{Ba}, \mathrm{Cd}, \mathrm{Cr}, \mathrm{Cu}, \mathrm{Hg}, \mathrm{Mo}, \mathrm{Ni}, \mathrm{Pb}, \mathrm{Sb}, \mathrm{Se}, \mathrm{Zn}$, cyanures totaux), les contaminants organiques (PCB (congénères 28, 52, 101, 118, 138, 180), HAP (16 composés), les butylétains (MBT, DBT, TBT), les hydrocarbure totaux et les BETEX (Benzène, Toluène, $o$-xylène, $\mathrm{m}+\mathrm{p}$ xylène, éthyl benzène).

Les données retenues concernent 7 ports de la région PACA (Arsenal (A), Bandol (BAND), Brégaillon (B), Cannes (CAN), St-Mandrier (STM), Vieux port de Marseille (VPM), Sanary-Lavandou (SALA) et 1 port de la région Bretagne (Guilvinec (GUIL)). Deux types de pré-traitements (dessablage, déshydratation) et 3 types de traitements (phosphatation, compostage, calcination), seuls ou combinés, ont été appliqués à ces sédiments à différents stades d'avancement du projet.

Enfin, cette étude statistique repose sur l'hypothèse que les échantillons bruts sont homogènes et représentatifs des sous échantillons qui ont pu être formés à partir d'un même sédiment brut.

\subsection{Stratégie des analyses statistiques}

Cette étude repose sur deux approches, le calcul du taux d'évolution relatif des concentrations des contaminants dans les différents échantillons après un ou plusieurs traitements et une analyse statistique factorielle (Analyse en Composante Principale, ACP).

Le taux d'évolution relatif des concentrations des contaminants a été calculé pour les différents échantillons afin d'évaluer l'évolution des concentrations des contaminants dans les sédiments après application d'un ou plusieurs pré-traitements/traitements et selon les différentes caractéristiques sédimentaires. Le taux d'évolution relatif est calculé par rapport à la contamination d'origine c'est-à-dire par rapport à la concentration des contaminants dans le sédiment brut, selon l'équation suivante:

Taux d'évolution relatif $=100 \times(([$ Brut $]-$ [après traitement $]) /[$ Brut $])$.

Ce taux relatif permet de mettre en évidence des tendances sur l'évolution des concentrations des contaminants en fonction des pré-traitements / traitements appliqués. Il permet également de confirmer la finalité d'un traitement par rapport aux différents contaminants et selon leur comportement chimique. Par exemple, après le dessablage qui permet de séparer la fraction sableuse de la fraction fine $(<63 \mu)$, les concentrations dans le sédiment dessablé devraient être plus importantes que dans le sédiment brut puisque la majeure partie des contaminants a plus d'affinité pour la fraction fine riche en matière organique et en argile (CHAPMAN et al., 1998). Le taux d'évolution relatif 
devrait donc être négatif. Au contraire, après la calcination qui permet de dégrader les composés organiques, les taux d'évolution relatifs pour le TBT ou les HAP devraient être positifs.

L'analyse en composante principale (ACP) a été utilisée pour discriminer les différents ports selon les caractéristiques physico-chimiques des sédiments et pour différencier et hiérarchiser les différents traitements selon les caractéristiques des sédiments. L'analyse factorielle regroupe un ensemble de méthodes statistiques descriptives qui permettent de présenter graphiquement le maximum d'informations contenues dans un tableau de données (LEBART et al., 2006).

L'analyse en composante principale (ACP) s'applique à des tableaux croisant des lignes, représentant les individus, et des colonnes représentant les variables. Dans cette étude, les individus sont les échantillons de sédiments des différents ports et les variables sont les propriétés physico-chimiques, les nutriments ou les contaminants chimiques. Le principe de l'ACP est de faire une représentation graphique des $\mathrm{n}$ individus dans un espace à $\mathrm{k}=2$ dimensions. La composante ayant la plus forte inertie est la composante la plus évidente et la plus facile à interpréter. Elle formera donc l'axe 1. Les résultats sont présentés sous la forme de deux graphiques: le cercle des corrélations des variables de base qui indique les corrélations entre les différentes variables et identifie les composantes principales 1 et 2 , et le plan factoriel des individus de bases formé par l'axe 1 et l'axe 2 dans lequel se répartissent les différents échantillons (SAPORTA, 2006). L'ACP a été réalisée avec l'extension UNIWIN du logiciel Statgraphics.

\subsection{Définition de la matrice statistique}

La matrice statistique qui a servi à cette étude est constituée de 86 échantillons et de 60 variables. Un codage a été défini pour pouvoir prendre en compte l'effet du traitement dans l'analyse statistique et pour labelliser les individus (échantillons de sédiments). Ce codage est basé sur l'origine de l'échantillon et sur le/les traitements appliqués au sédiment constituant l'échantillon. Le codage respecte l'ordre chronologique lorsque plusieurs traitements sont appliqués. Les sédiments ont été labellisés selon les codes donnés entre parenthèses dans le paragraphe précédent décrivant les ports étudiés. Pour les traitements, des chiffres de 0 à 5 ont été utilisés et sont définis de la façon suivante : Pas de traitement (sédiment brut): 0 ; Dessablage: 1; Déshydratation: 2; Phosphatation : 3 ; Compostage de 1/2/3/4/ 5/ 6 ; mois : 4a/4b/4c/4d/4e/ 4f; Calcination : 5 . 


\section{Résultats et Discussion}

3.1 Discrimination des échantillons de sédiments prélevés

Une analyse en composantes principales a été réalisée afin de pouvoir discriminer les différents ports étudiés selon leurs caractéristiques chimiques et leurs concentrations en contaminants. Le cercle des corrélations des variables de base et le plan factoriel des individus de base sont présentés figures 2 et 3 respectivement.

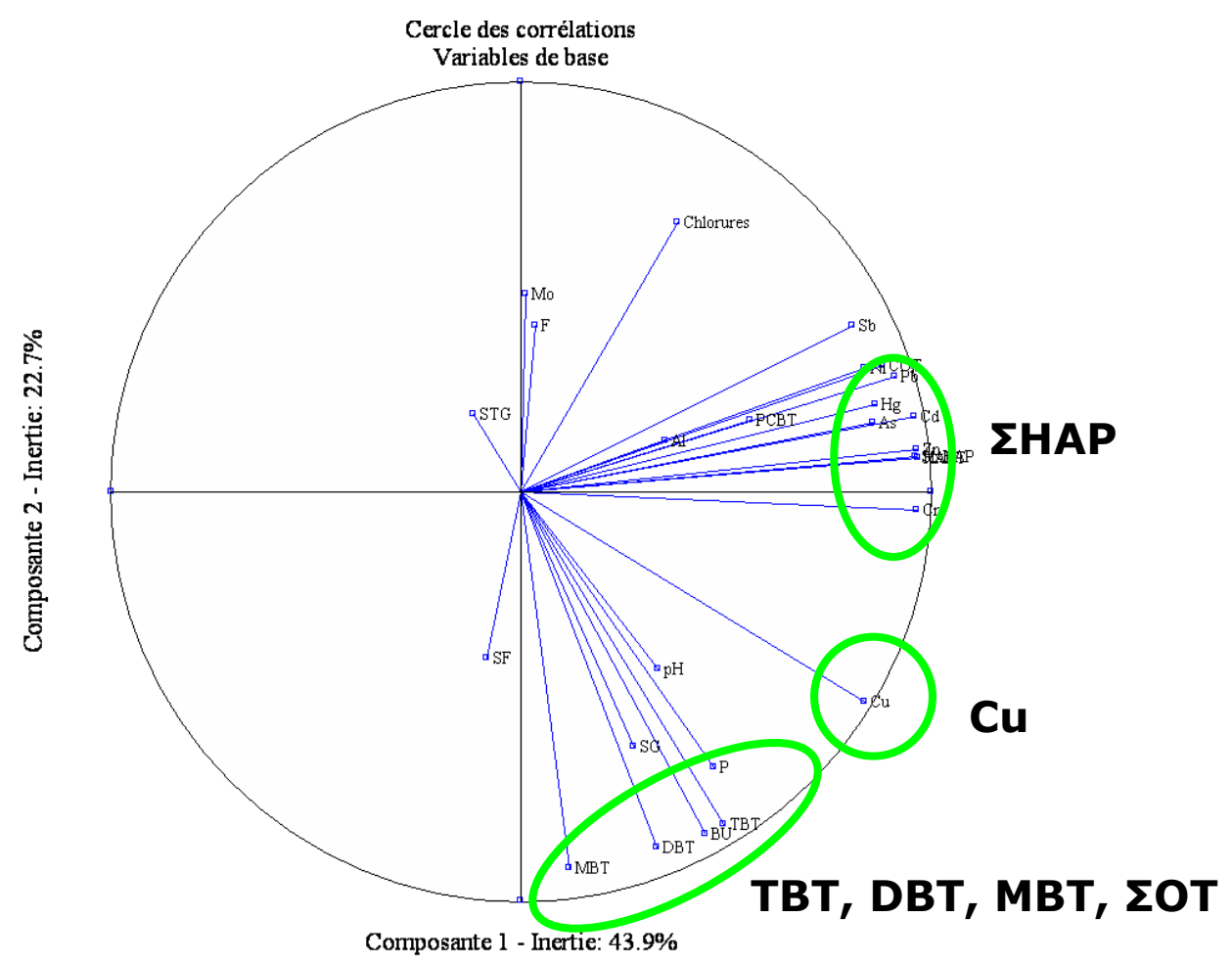

Figure 2. Cercle des corrélations des variables de base relatives aux sédiments bruts.

Le cercle des corrélations des variables de base montre que la composante principale 1 représente des corrélations essentiellement positives entre toutes les variables contribuant à cet axe. Cette composante représente donc un niveau global de pollution en métaux et contaminants organiques (sauf organoétains) croissant de gauche à droite. D'autre part, les concentrations en MBT, DBT, TBT et $\Sigma \mathrm{OT}$ ( $\Sigma \mathrm{OT}=$ somme des organoétains $=\mathrm{MBT}+\mathrm{DBT}+\mathrm{TBT}$ ) sont les variables qui ont la plus forte contribution dans la formation de la composante principale 2. Cette composante principale 2 représente la contamination en organoétains. 


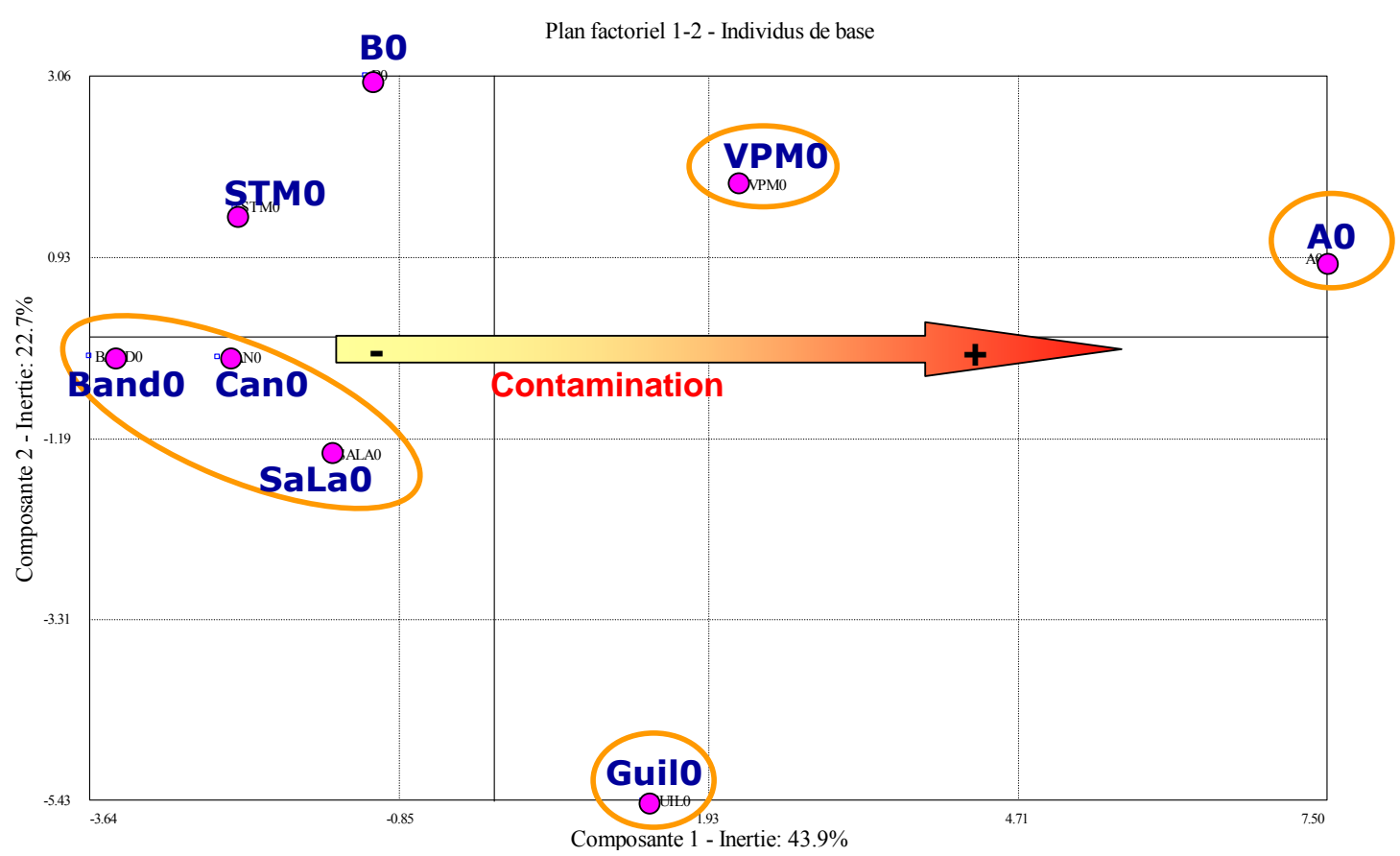

Figure 3. Plan factoriel des individus de base relatifs aux sédiments bruts.

Le plan factoriel des individus de base (figure 3) montre une répartition des échantillons le long de l'axe 1 selon leur niveau de pollution global en métaux et HAP et, de part et d'autre de l'axe 2, selon leur niveau de contamination en organoétains. Deux ports, celui de l'Arsenal (A0) et celui du Guilvinec (GUIL 0), se distinguent des autres ports et sont caractérisés par une pollution importante. Le port d'Arsenal contient une forte contamination en métaux, HAP et PCB. Le port du Guilvinec est caractérisé par une forte contamination en TBT. Les autres ports peuvent être considérés comme faiblement à moyennement contaminés et peuvent être classés selon l'ordre croissant de contamination suivant: Bandol, Cannes, Saint-Mandrier, Sanary-Lavandou, Brégaillon et le Vieux Port de Marseille.

\subsection{Discrimination et hiérarchisation des traitements}

Le plan factoriel des individus de base obtenu après application de l'ACP est présenté figure 4.

La figure 4 montre des niveaux globaux de pollution très différents selon les ports et une forte différence entre les effets des traitements. Quatre groupes de ports peuvent être discriminés selon leur état de pollution. Le groupe Arsenal est considéré comme le plus contaminé et le groupe dit "Autres ports" comme le moins contaminé.

Au niveau des traitements, il semble que le compostage soit un traitement efficace pour diminuer la charge polluante des sédiments bruts et dessablés de l'Arsenal et du Guilvinec. Les effets positifs des différents traitements sur les sédiments faiblement à moyennement contaminés sont moins marqués. 


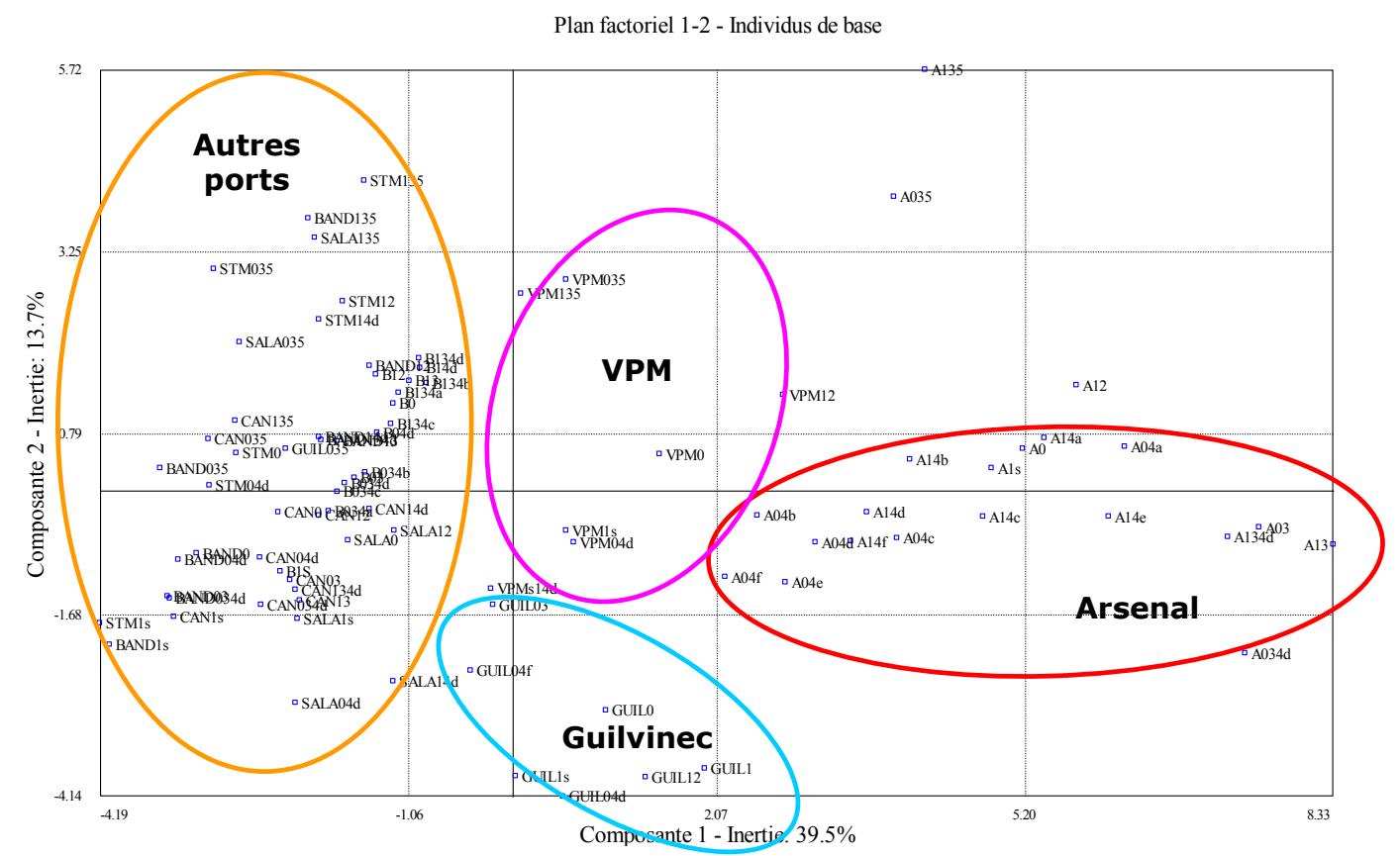

Figure 4. Plan factoriel des individus de base relatifs à l'ensemble des échantillons.

\subsection{Taux d'évolution relatif des concentrations de certains contaminants}

Le calcul de ce taux permet de mettre en évidence l'incidence d'un ou plusieurs traitements sur l'évolution de la charge polluante contenue dans un sédiment. Un taux d'évolution positif traduit une réduction de la concentration en contaminant entre le matériau brut et le matériau traité et inversement. Un taux d'évolution proche de zéro montrera que la concentration en contaminant est restée stable. Toutefois, en fonction des contaminants considérés et $\mathrm{du} / \mathrm{des}$ traitements appliqués, la valeur positive ou négative du taux d'évolution va être fonction de la finalité du traitement.

Pour les huit ports considérés, les taux d'évolution ont été calculés pour trois types de contaminants (métalliques, organométalliques, organiques) d'importance dans le contexte d'un dragage, le TBT, les HAP et le cuivre. Ces contaminants accumulés dans les sédiments portuaires proviennent d'activités anthropiques présentes ou passées. Le TBT et le cuivre proviennent principalement des peintures anti-salissures dans lesquelles ils sont utilisés pour leurs propriétés biocides. Le TBT est interdit en France depuis 1982 sur les navires de moins de $25 \mathrm{~m}$ et depuis le $1^{\text {er }}$ janvier 2008 pour tous les navires entrant dans un port européen, en raison des effets délétères qu'il occasionne chez les organismes aquatiques à des concentrations très faibles dans l'eau inférieures au ng $\mathrm{L}^{-1}$. Dans les années 80 , le TBT a été petit à petit été remplacé par l'oxyde de cuivre $(\mathrm{Cu} 2 \mathrm{O})$ comme matière active des peintures anti-salissures, c'est la raison pour laquelle on mesure des concentrations élevées de cuivre dans les sédiments portuaires (ALZIEU, 2000). Les HAP, quant à eux, proviennent principalement de la combustion 
des essences et des déchets industriels et urbains via les apports continentaux (BAUMARD et al., 1998).

Les résultats obtenus sont présentés sous forme de graphiques qui montrent l'évolution du taux d'évolution relatif en fonction de l'origine des différents sédiments.

Dans le cas du TBT, les taux d'évolution obtenu en fonction du/des traitement(s) appliqués et en fonction de l'origine des sédiments est présenté sur la figure 5.

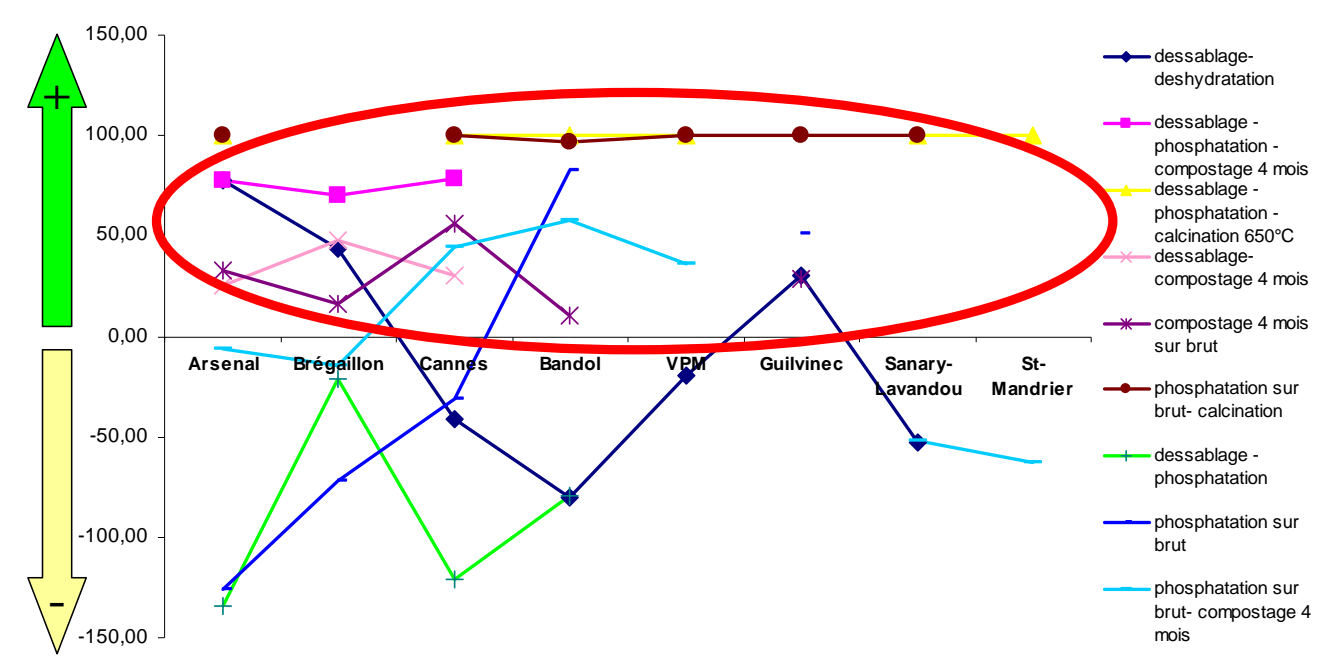

Figure 5. Discrimination des traitements en fonction de l'évolution du taux de réduction du TBT.

Le taux d'évolution du TBT est positif lorsque des traitements sont appliqués seuls ou bien en combinaison avec d'autres traitements. Les traitements seuls ayant un effet positif sur la réduction de la concentration en TBT sont le dessablage (pour la fraction sableuse du sédiment), la calcination à $650^{\circ} \mathrm{C}$ et le compostage pendant une période de 4 mois. Le dessablage est un pré-traitement efficace car le TBT a tendance à se fixer sur les particules fines qui sont généralement riches en matière organique (SARRADIN, 1995). Une fois séparé de la fraction fine, le sable est donc la fraction sédimentaire dans laquelle la concentration en TBT est généralement faible ou inexistante. L'efficacité de la calcination à dégrader le TBT s'explique par le fait que le TBT est une molécule organique qui est détruite sous l'action d'une forte température (CRAIG, 1986). Enfin, l'effet positif du compostage s'explique par une oxydation de la masse sédimentaire et le développement d'une activité microbiologique qui participe à la dégradation du TBT (AMOUROUX et al., 2000). L'action des UV favorise également la dégradation du TBT (DONARD et al., 2001). Ces traitements combinés entre eux tels que le dessablage et le compostage ou le dessablage et la calcination ont également un effet positif sur la diminution de la charge polluante en TBT. Par ailleurs, si on considère la phosphatation, la figure 5 montre des taux d'évolution très différents selon le type de 
sédiment. En effet, dans le cas de la phosphatation appliquée au sédiment brut, le taux d'évolution est positif pour Arsenal, alors que pour Bandol et Cannes, le taux est négatif. Dans le cas d'un taux d'évolution négatif, la phosphatation favoriserait la rétention du TBT. Cela peut s'expliquer par le fait que le TBT, sous forme cationique, pourrait être piégé dans des phosphates calciques et de ce fait, être stabilisé. Toutefois, seuls des tests supplémentaires incluant une meilleure connaissance de la mobilité permettraient de conclure. Ainsi, une étude récente a montré que ce traitement aurait tendance à inhiber la dégradation du TBT en composés moins toxiques (DBT et MBT) mais également qu'un faible pourcentage (inférieur à $0,1 \%$ ) de ce composé contenu dans le sédiment pouvait être remobilisé (SEBY \& BENOIT-BONNEMASON, 2008 ; SEBY et al., 2009). A partir de ces résultats, il semble que l'efficacité de la phosphatation soit encore à démontrer pour le TBT et que ce processus dépend des caractéristiques physico-chimiques des sédiments.

La figure 6 présente l'évolution du taux de réduction des HAP selon le/les traitement(s) appliqués en fonction de l'origine des sédiments.

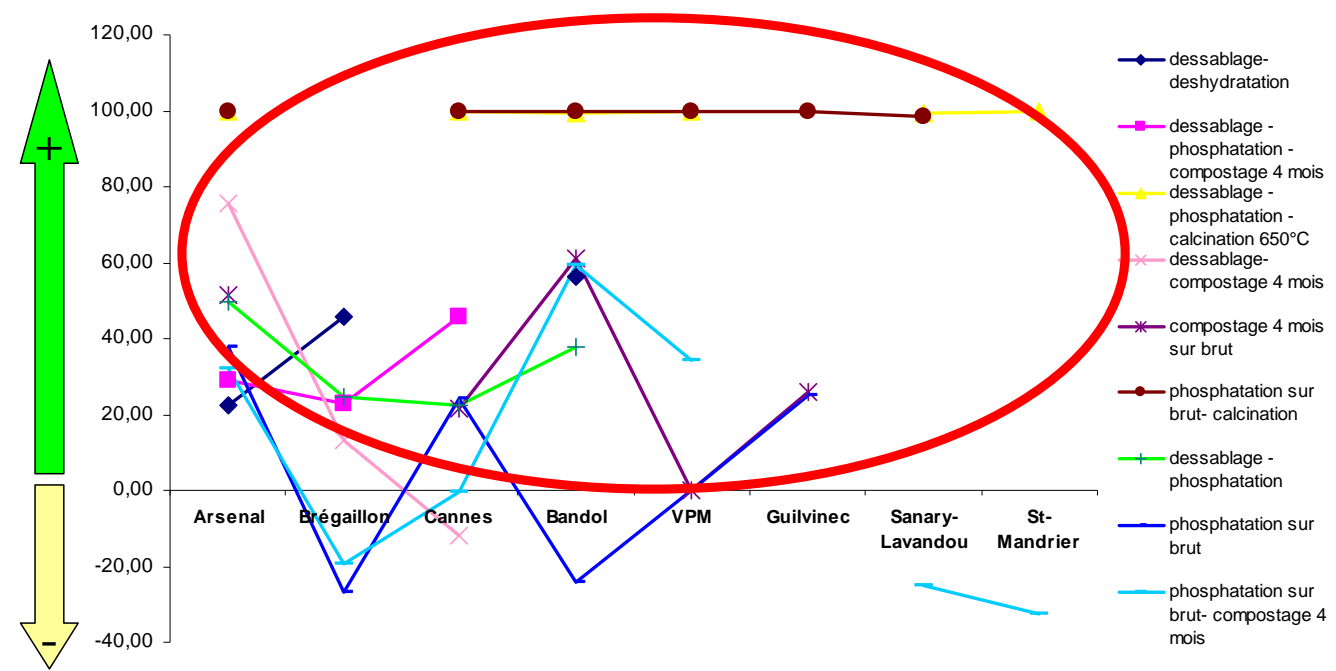

Figure 6. Discrimination des traitements en fonction de l'évolution du taux de réduction des HAP.

Dans l'ensemble, le taux d'évolution relatif des HAP est positif d'une part pour des traitements appliqués seuls ou ceux qui sont combinés à du dessablage comme la phosphatation, la déshydratation, le compostage pendant 4 mois ou encore la calcination à $650{ }^{\circ} \mathrm{C}$. D'autres combinaisons de traitements telles que la phosphatation et le compostage (4 mois) ont un effet positif sur la diminution de la charge polluante en HAP. Excepté pour le compostage, l'efficacité de ces traitements appliqués seuls ou plus généralement à du dessablage peut s'expliquer par une augmentation de la température qui favorise la dégradation des HAP (TRONCZYNSKI et al., 2005). Dans 
le cas du compostage, l'action des UV associée à une activité microbiologique dégrade ces composés (GAN et al., 2009). En revanche, aucun effet remarquable n'a pu être mis en évidence pour la déshydratation appliquée sur les sédiments bruts.

L'évolution du taux de réduction du cuivre selon le/les traitement(s) appliqués et en fonction de l'origine des sédiments est présenté figure 7.

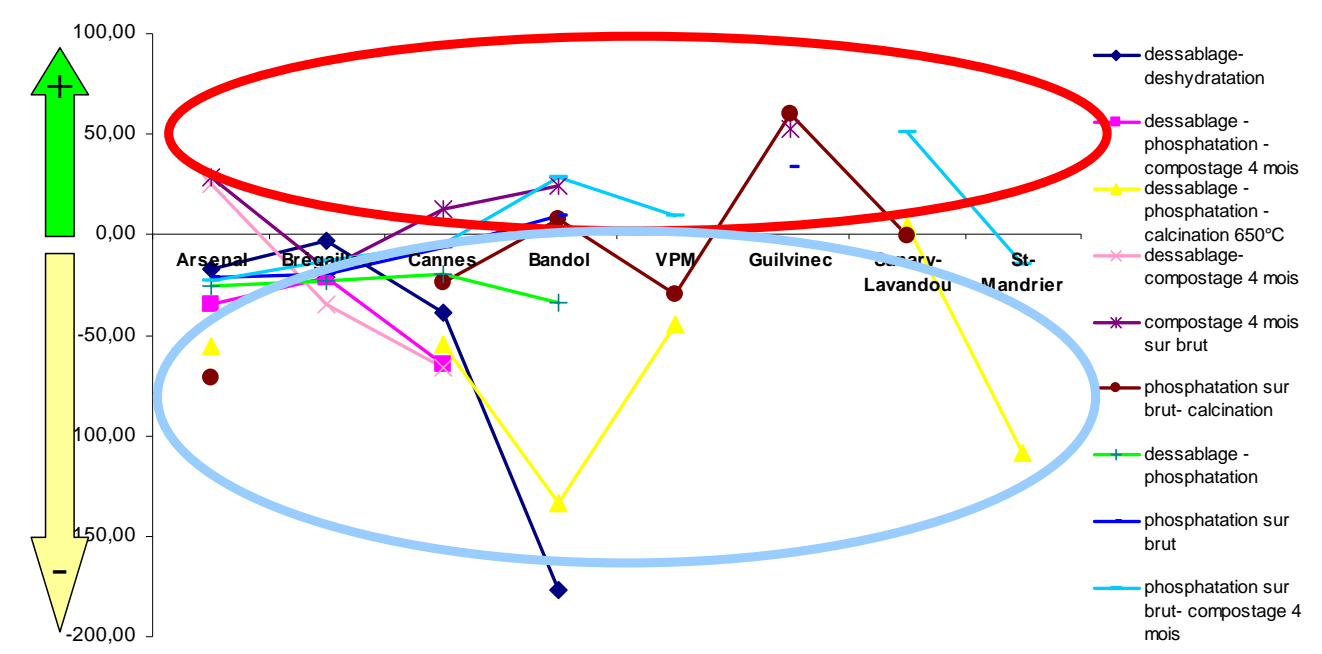

Figure 7. Discrimination des traitements en fonction de l'évolution du taux de réduction du cuivre.

D’une manière générale, les taux d'évolution du cuivre sont négatifs ou proches de zéro, ce qui traduit une concentration en cuivre après traitement supérieure à la concentration avant traitement. Cette tendance est observée notamment dans la fraction fine après dessablage et déshydratation et pour la majorité des échantillons après traitement par phosphatation combiné ou non avec un autre traitement. Des taux proches de zéro peuvent montrer que la phosphatation ne permet pas de piéger le cuivre principalement dans des phosphates calciques comme par exemple des hydroxyapatites. Ce principe d'inertage ainsi que la stabilité chimique et thermique des composés formés a été mise en évidence par plusieurs études (CRANNELL et al., 2000 ; BOURNONVILLE et al., 2004). Dans les sédiments étudiés, seules des études de mobilité permettraient de s'assurer de l'efficacité de l'inertage. L'étude de SEBY \& BENOIT-BONNEMASON (2008) sur l'évolution des métaux et de leurs formes chimiques dans des vases portuaires stockées à terre, a montré qu'un faible pourcentage du cuivre $(0,05 \%)$ contenu dans le sédiment brut phosphaté était lixivié au bout de 2 mois. Même si les taux de lixiviation sont très faibles, ces résultats montre l'importance de compléter les analyses de caractérisation chimique des sédiments par des analyses permettant de mettre en évidence la forme chimique du contaminant et la façon dont il est lié à la 
structure sédimentaire (par exemple, tests de lixiviation, extractions séquentielles, analyses par les techniques du solide (XPS, XANES, etc.)).

\section{Conclusion}

Une analyse statistique a été menée à partir de la base de données issue du projet SEDI.MAR.D 83 afin de discriminer les ports selon leur contamination et hiérarchiser les différentes filières de traitement. En ce qui concerne le niveau de contamination, deux ports peuvent être différenciés du fait d'une pollution importante, le port d'Arsenal caractérisé par une forte contamination en métaux, HAP et PCB et le port du Guilvinec, particulièrement marqué par une forte contamination en TBT. Comparativement, les autres ports sont faiblement à moyennement contaminés sans prédominance d'un contaminant par rapport à un autre. Une forte différence entre les effets des traitements a également été observée. Elle est d'autant moins marquée que le sédiment est peu ou faiblement contaminé.

Ainsi, le compostage pendant une période de 4 mois semble être le traitement le plus efficace pour les sédiments bruts et dessablés s'ils sont relativement contaminés. Cette tendance est observée notamment pour les sédiments provenant des ports d'Arsenal et du Guilvinec dont le niveau de contamination est assez élevé.

Par ailleurs, l'incidence d'un ou plusieurs traitements sur l'évolution de la charge polluante contenue dans un sédiment varie selon les différents types de contaminants (métalliques, organométalliques, organiques). En effet, pour un sédiment contaminé en composés organiques et/ou en composés organométalliques (HAP et TBT), les traitements par calcination $\left(650{ }^{\circ} \mathrm{C}\right)$ et compostage (importance de la durée) vont permettre un abattement de la concentration par dégradation de ces composés selon différents processus physico-chimiques et/ou biologiques (action d'une très haute température pour la calcination, activité bactérienne, la volatilisation, la photochimie ou encore l'oxydo-réduction pour le compostage). En ce qui concerne le cuivre, le compostage ne semble pas avoir d'effet significatif sur l'abattement des concentrations, très probablement $\mathrm{du}$ fait de la très grande affinité des éléments métalliques pour la fraction fine et des conditions physico-chimiques du sédiment qui favorisent la formation d'oxydes stables en présence d'oxygène et une complexation avec la matière organique. La phosphatation et la calcination favorisent l'inertage du sédiment par piégeage du cuivre dans la masse sédimentaire. Il semble toutefois judicieux de réaliser des analyses complémentaires (test de lixiviation, extractions séquentielles) afin de mettre en évidence la part remobilisable qui rend le contaminant biodisponible et de fait augmente la toxicité du sédiment.

\section{Références bibliographiques}

ALZIEU C. (2000). Environmental impact of TBT: the French experience. The Science of the Total Environment, Vol. 258, pp 99-102. doi:10.1016/S0048-9697(00)00510-6 
AMOUROUX D., TESSIER E., DONARD O.F.X. (2000). Volatilization of organotin compounds from estuarine and coastal environments. Environmental Science \& Technology, Vol. 34, pp 988-995. doi:10.1021/es981025p

AQUA J.-L., BOISSERY P., ALZIEU C. (2009). Un programme opérationnel d'identification des modes de gestion durable des sédiments marins : le projet SEDI.MAR.D. 83, Revue Paralia, Vol. 2, pp s1.1-s1.12. doi:10.5150/revue-paralia.2009.s01

BAUMARD P., BUDZINSKI H., MICHON Q., GARRIGUES P., BURGEOT T., BELLOCQ J. (1998). Origin and bioavalaibility of PAHs in the Mediterranean Sea from mussel and sediment records. Esturaine, Coastal and shelf science, Vol. 47, pp 77-90. doi:10.1006/ecss.1998.0337

BENOIT C. (2005). Biogéochimie et enregistrement sédimentaire des composés organostannques dans les sédiments du Bassin d'Arcachon. Thèse de l'Université de Bordeaux 1, $207 \mathrm{p}$.

BOURNONVILLE B., NZIHOU A., SHARROCK P., DEPELSENAIRE G. (2004). Stabilisation of heavy metal containing dusts by reaction with phosphoric acid: study of the reactivity of fly ash. Journal of Hazardous Materials, B116, pp. 65-74. doi:10.1016/j.jhazmat.2004.07.021

Bulletin officiel du ministère chargé des transports $n^{\circ} 2000 / 16$ p. 74-81, Circulaire $n^{\circ}$ 2000-62 du 14 juin 2000 relative aux conditions d'utilisation du référentiel de qualité des sédiments marins ou estuariens en milieu naturel ou portuaire défini par l'arrêté ministériel. Disponible sur http://www.legifrance.gouv.fr/

CALMANO W., FORSTNER U. (1996). Sediments and toxic substances. Springer Verlag, Berlin, 335 p.

CHAPMAN P.M., WANG F., JANSEEN C., PERSOONE G., ALLEN H.E. (1998). Ecotoxicology of metals in aquatics sediments: binding and release, bioavalability, risk asessment and remediation. Canadian Journal of Fisheries and aquatic sciences, Vol. 55(10), pp 2221-2243. doi:10.1139/f98-145

CRAIG P.J. (1986) Organometallic compounds in the environment. Longman, London, $415 \mathrm{p}$.

CRANNELL B.S., EIGHMY T.T., KRZANOWSKI J.E., EUSDEN JR. J.D., SHAW E.L., FRANCIS C.A. (2000). Heavy metal stabilization in municipal solid waste combustion bottom ash using soluble phosphate. Waste Management, Vol. 20, pp 135-148. doi:10.1016/S0956-053X(99)00312-8

DELORT E., GRODEMANGE D. (2006). Du dragage, des traitements et des filières de valorisation de sédiments portuaires méditerranéens. Contexte réglementaire, filières envisageables en PACA et dans le Var, chaîne de prétraitement et de traitement, scénarii pour le dragage et la valorisation des sédiments. Note technique, Rapport intermédiaire, $170 \mathrm{p}$. 
DONARD O.F.X., LESPES G., AMOUROUX D., MORABITO R. (2001). Organotin compounds in the environment: still a most critical issue. In: Trace element speciation for environment, food and health. Cornelis R., Crews H., Donard O.F.X., Ebdon L., Quevauviller P., Eds. Royal Society of Chemistry, 260 p. doi:10.1039/9781847552204-00142 GAN S., LAU E.V., NG H.K. (2009). Remediation of soils contaminated with polycyclic aromatic hydrocarbons (PAHs). Journal of Hazardous Materials, Vol. 172, pp 532-549. doi:10.1016/j.jhazmat.2009.07.118

HOCH M. (2001). Organotin compounds in the environment: an overview. Applied Geochemical, Vol. 16, pp 719-743. doi:10.1016/S0883-2927(00)00067-6

JO n ${ }^{\circ} 184$ du 10 août 200012415 texte $n^{\circ}$ 24, Arrêté du 14/06/00 relatif aux niveaux de référence à prendre en compte lors d'une analyse de sédiments marins ou estuariens présents en milieu naturel ou portuaire. Disponible sur http://www.legifrance.gouv.fr/ LANDRUM P.F., ROBBINS J.A. (1990). Bioavailability of sediment-associated contaminants to benthic invertebrates. In Sediments: chemistry and toxicity of in-place pollutants. Eds Baudo. R, Giesy J.P., Muntau H., Lewis publishers, Michigan. Vol. 8, pp 237-263.

LEBART L., MORINEAU A., PIRON M. (2006) Statistique exploratoire multidimensionnelle, Dunod, Paris.

MAROT F. (1997). Caractérisation et traitement de sédiments de dragage contenant des polluants métalliques. Thèse de l'Université du Havre, $330 \mathrm{p}$.

QINGMAN L., SHUPING B., GUOLIANG J. (2003). Determination of strongly reducing substances in sediment. Environmental Science \& Technology, Vol. 37, pp 5527-5731.

RULKENS W. (2005). Introduction to the treatment of polluted sediments. Review in Environmental Sciences and Bio/Technology, Vol. 4, pp 213-221. doi:10.1007/s11157-0052167-6

RIOS L. M, MOORE C., JONES P.R. (2007). Persistent organic pollutants carried by synthetic polymers in the ocean environment. Marine Pollution Bulletin, Vol. 54, pp 1230-1237. doi:10.1016/j.marpolbul.2007.03.022

SANNIER L., LEVACHER D., JOURDAN M. (2009). Approche économique et validation de méthodesde traitements aux liants hydrauliques de sédiments marins contaminés. Revue Paralia, Vol. 2, pp s2.1-s2.15. doi:10.5150/revue-paralia.2009.s02

SAPORTA G. (2006). Probabilités, analyses des données et statistique. Technip, Paris. SARRADIN P.M., LAPAQUELLERIE Y., ASTRUC A., LATOUCHE C., ASTRUC M. (1995). Long term behaviour and degradation kinetics of tributyltin in a marina sediment, The Science of the Total Environment, Vol. 170, pp 59-70. doi:10.1016/00489697(95)04561-E

SEBY F., BENOIT-BONNEMASON C. (2007). Evolution des métaux et de leurs formes chimiques dans des vases portuaires dans le cadre d'un stockage à terre, Rapport d'étude, 49 p. 
SEBY F., BENOIT-BONNEMASON C., TESSIER E., ALZIEU C., AQUA J.-L., SANNIER L., DONARD O.F.-X. (2009). Etude de l'évolution des formes chimiques des métaux dans des sédiments marins dragués stockés à terre, Revue Paralia, Vol. 2, pp s3.1-s3.12. doi:10.5150/revue-paralia.2009.s03

TRONCZYNSKI J, MUNSCHY C, HEAS-MOISAN K, GUIOT N, TRUQUET I, (2005). Analyses de contaminants organiques (PCB, OCP, HAP) dans les organismes marins. Coll. Méthodes d'analyse en milieu marin, $44 \mathrm{p}$.

VERMEULEN J, GROTENHUIS J.C.T., JOZIASSE J., RULKENS W.H. (2003). Ripening of clayey dredged sediments during temporary unplanned disposal: a bioremediation technique. Journal of soils and sediments, Vol. 3, $\mathrm{n}^{\circ}$ 1, pp 49-59. doi:10.1007/BF02989469

WRIGHT P., MASONU C.F. (1998). Spatial and seasonal variation in heavy metals in the sediments and biota of two adjacent estuaries, the Orwell and the Stour, in eastern England. The Science of the Total Environment, Vol. 226, pp 139-156. doi:10.1016/S00489697(98)00383-0 


\title{
Statistical analysis of data obtained on sediments treated on the test site SEDI.MAR.D 83
}

\author{
Christelle BENOIT-BONNEMASON ${ }^{1}$, Fabienne SEBY ${ }^{1}$, \\ Jean-Christophe TURLOT ${ }^{2}$, Mickael JOURDAN ${ }^{3}$, Claude ALZIEU ${ }^{3}$, \\ Jean-Luc AQUA ${ }^{3}$, Laurent SANNIER ${ }^{3}$, Olivier F.X. DONARD ${ }^{4}$
}

1. UT2A, Hélioparc Pau-Pyrénées, 2 Av du Pdt Angot, 64053 Pau cedex 9, France. christelle.benoit@univ-pau.fr

2. LMA/ UMR CNRS 5142, UPPA, Av de l'Université, 64000 Pau, France

3. Conseil Général du Var, Direction des Ports, 390 Av des Lices, BP1303, 83076 Toulon cedex, France.

4. LCABIE / IPREM, UMR CNRS/UPPA 5254, Hélioparc Pau-Pyrénées, 2 Av du Pdt Angot, 64053 Pau cedex 9, France.

Translated version not provided by the authors

Version traduite non fournie par les auteurs 
3.18 : Revue Paralia - Vol. 5 (2012) 